\title{
Phase Separation in Binary Fluid Mixtures with Continuously Ramped Temperature
}

\author{
By M. E. Cates ${ }^{1}$, J. Vollmer ${ }^{2}$, A. Wagner ${ }^{1,3}$, D. Vollmer ${ }^{1,4}$ \\ ${ }^{1}$ School of Physics, University of Edinburgh, King's Buildings, Edinburgh EH9 \\ 3JZ, UK \\ ${ }^{2}$ Max-Planck Institute for Polymer Research, Ackermannweg 10, 55128 Mainz, \\ Germany \\ ${ }^{3}$ Department of Physics, North Dakota State University, Fargo, ND 58105, USA \\ ${ }^{4}$ Institute for Physical Chemistry, University of Mainz, Welder-Weg 11, 55099 \\ Mainz, Germany
}

\begin{abstract}
We consider the demixing of a binary fluid mixture, under gravity, which is steadily driven into a two phase region by slowly ramping the temperature. We assume, as a first approximation, that the system remains spatially isothermal, and examine the interplay of two competing nonlinearities. One of these arises because the supersaturation is greatest far from the meniscus, creating inversions of the density which can lead to fluid motion; although isothermal, this is somewhat like the Bénard problem (a single-phase fluid heated from below). The other is the intrinsic diffusive instability which results either in nucleation or in spinodal decomposition at large supersaturations. Experimental results on a simple binary mixture show interesting oscillations in heat capacity and optical properties for a wide range of ramp parameters. We argue that these oscillations arise under conditions where both nonlinearities are important.
\end{abstract}

Keywords: demixing, gravity, temperature ramp, binary fluids

\section{Introduction}

If a binary fluid, made of two species that are miscible at high temperature, is suddenly quenched into the two-phase region, it starts to demix. Depending on composition and quench depth, the mechanism for demixing and subsequent domain growth is either spinodal decomposition (amplification of small compositional fluctuations, at first by diffusion and then by fluid motion driven by interfacial tension) or nucleation and diffusive growth of small droplets of one phase in the other (Bray 1994, 2000; Onuki 2002). Unless the two fluids have exactly matched densities, gravity eventually takes over, once the domain (or droplet) size becomes comparable to a suitably defined capillary length (Onuki 2002). The details of this gravitational stage are not fully understood and involve interesting new physics such as 'lane formation' (Chan \& Goldberg 1975, Aarts et al. 2002). Nonetheless, it is observed that, once gravity does intervene, fluids separate relatively rapidly leading finally to a flat horizontal meniscus between phases. 
In the natural world, and even in most laboratory settings, rapid temperature changes are a relatively rare occurrence. The opposite case of a very slow temperature ramp, though equally idealised, is arguably closer to most everyday instances of phase separation, and it is certainly important to understand this limiting case. However, the physics is more complex, because the system is now subject to continuous driving, as opposed to being displaced instantaneously from equilibrium, and allowed to relax back towards it.

If the ramp rate $d T / d t$ is small enough, one can hope that the time taken for heat to diffuse across the sample is small compared to other time scales of interest $\dagger$. If so, this allows us to treat the system as isothermal at any instant; for simplicity we shall do this here, although in the experiments that motivate this work, the spatial gradients of temperature may not in fact be negligible. Neglecting all such gradients reduces the problem from a double-diffusive one (Brandt \& Fernando 1995), where diffusion of heat and composition are both important, into one involving compositional diffusion only.

Despite this the problem is still 'doubly nonlinear'. The first nonlinearity is standard, and arises from the coupling between density differences (caused by composition deviations) to gravity. But the fact that the system can show phase separation requires that, even without this coupling, compositional diffusion is already a highly nonlinear process: a linearized diffusion equation obviously cannot yield spinodal decomposition and/or nucleation of droplets. The interplay of these two nonlinearities, for the case of a slow isothermal temperature ramp in a binary fluid system undergoing phase separation, is addressed in this paper.

\section{Experimental motivation}

Vollmer et al. (2002) reported differential calorimetric studies of the phase separation of a binary fluid mixture comprising water and 2 butoxy ethanol, also called $\mathrm{C}_{4} \mathrm{E}_{1}$, at low ramp rates (a few Kelvins per hour). The system has a lower consolute point and therefore demixes when heated up. The experiments revealed a quite unexpected effect: after an incubation period during which the phase boundary is first crossed and a meniscus forms, the observed heat capacity passes through a series of oscillations (typically about six in number) before eventually decaying to a smooth curve (Figure 1, upper left). The period and overall duration of the oscillations depends on heating rate; it also depends on sample geometry, and on the composition of the initial state.

An experiment in which heating was stopped for several hours midway through the oscillations (Figure 1, upper right) showed that these resumed when heating was recommenced, with only a small time delay; their period was not affected. Moreover, the eventual decay of the oscillations occurred exactly as though no interruption had occurred. These observations establish that there is little inertia involved in the process (since the interruption brings all fluid motion to a halt), and also suggest that the final decay of the oscillations is intrinsic, rather than stemming (for example) from a gradual loss of coherence in different parts of the system. (The recommencement of heating after a long break would presumably restore any required coherence.)

$\dagger$ This time $\tau_{h}$ obeys $\Lambda_{y}^{2} \sim \kappa_{T} \tau_{h}$ where $\Lambda_{y}$ is the smallest dimension of the sample cell (its thickness) and $\kappa_{T}$ the thermal diffusivity. 

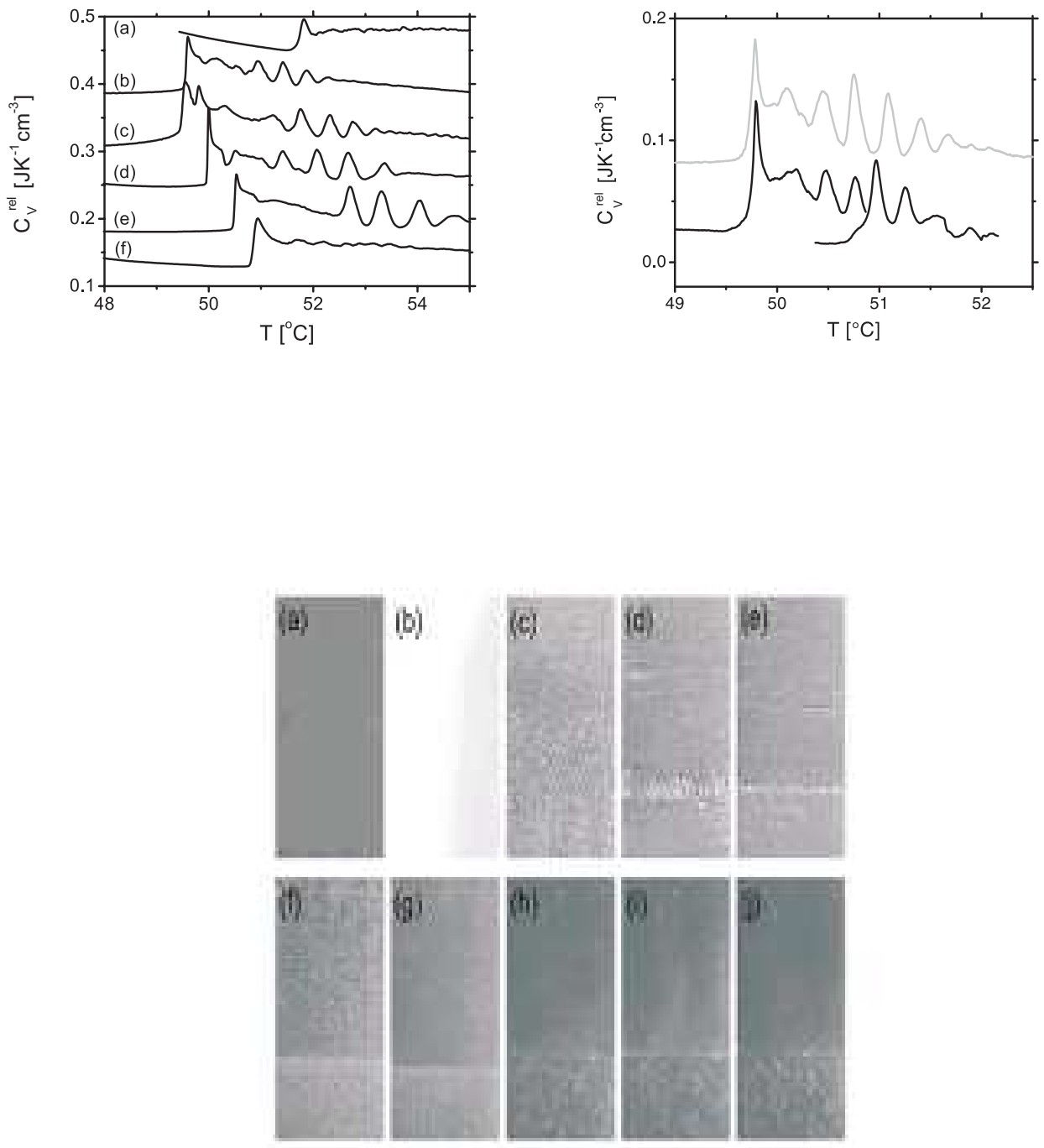

Figure 1. Heat capacity measurements and videomicroscopy for mixtures of $\mathrm{C}_{4} \mathrm{E}_{1}$ and water. (top left) Apparent heat capacity of mixtures of various initial compositions [(a) 0.18; (b) 0.25 ; (c) 0.35 ; (d) 0.39 ; (e) 0.43 ; (f) $0.44 \%$ volume fractionC $\mathrm{C}_{4} \mathrm{E}_{1}$ ] that undergo a temperature ramp of $2 \mathrm{~K} /$ hour. (top right) Comparison of a scan $\left(0.4 \mathrm{C}_{4} \mathrm{E}_{1}\right.$ by volume, $0.8 \mathrm{~K} /$ hour ramp rate) with one where the heating was stopped after the first three oscillations and resumed at a later time (black). (bottom) Videomicroscopy sequence for a heating rate of $10 \mathrm{~K} /$ hour and an initial composition of $32 \% \mathrm{C}_{4} \mathrm{E}_{1}$ by volume. The temperatures $\left({ }^{\circ} \mathrm{C}\right.$ ) are (a) 48.8, (b) 50.5, (c) 51.1, (d) 51.2, (e) 51.3, (f) 52.4, (g) 53.4 , (h) 57.9, (i) 58.8, (j) 59.5. The first oscillation in the sequence is visible in the final three frames. In frame (b) the sample is white. (The upper left and bottom figures are reproduced from Vollmer et al. (2002).) 
By observing the upper phase present in these samples using contrast enhanced videomicroscopy (Figure 1, bottom) the oscillations were associated with the following cycle. One observes an onset of high turbidity as large numbers of small droplets are suddenly created; these coarsen, and are soon cleared from the system by gravity. Bulk fluid motion is visible during this sedimentation stage; the system is then left in a quiet, transparent state with few droplets present. Soon after, the process repeats. In the quiet interludes between episodes of droplet formation, it is observed that fluid motion continues.

\section{Theoretical Considerations}

\section{(a) Equations of motion}

We introduce a minimal model which is not strictly realizable in experiment (although it can more nearly be achieved in computer simulation; see Vollmer et al., unpublished). This model addresses an isothermal, thermodynamically symmetric binary fluid, whose kinematic viscosity $\nu$ (i.e., the diffusivity of momentum) is independent of composition and temperature, and whose mass density $\rho$ depends on the composition variable $\phi$ through a temperature independent parameter $\alpha=$ $\rho^{-1} \mathrm{~d} \rho / \mathrm{d} \phi$. The equilibrium phase diagram is symmetric about $\phi=\phi_{c}=0$, with the compositions of the two coexisting phases (at the binodal) obeying $\phi= \pm \phi_{0}(T)$. The fluid is taken to be incompressible $(\nabla \cdot \mathbf{u}=0)$.

The Navier Stokes equation for the fluid velocity $\mathbf{u}$ may then be written

$$
\frac{\partial \mathbf{u}}{\partial t}+\mathbf{u} \cdot \nabla \mathbf{u}+\alpha \mathbf{u}\left(\frac{\partial \hat{\phi}}{\partial t}+\mathbf{u} \cdot \nabla \hat{\phi}\right)=\nu \nabla^{2} \mathbf{u}+\alpha g \phi_{0} \hat{\phi} \nabla z-\nabla(p / \rho+g z)
$$

Here $g$ denotes gravity, acting towards $-z ; p$ is the fluid pressure; and $\hat{\phi}=\left(\phi_{0}-\right.$ $\phi) / \phi_{0}$ is a relative supersaturation that measures the deviation of the local composition from the equilibrium value $\phi_{0}$. This holds in regions of positive $\phi$; otherwise the sign of $\hat{\phi}$ is reversed. (Note that a state of zero $\hat{\phi}$ can describe two phases at equilibrium, with a sharp meniscus in between.) In what follows, we shall assume that the effects of composition on fluid density (proportional to $\alpha$ ) mainly enter through the term in $g$ on the right hand side: this term represents a buoyancy effect in which local composition affects the body force acting directly on the fluid. The other place that $\alpha$ enters, on the left of the equation, represents the fact that changes in mass density caused by varying composition alter the acceleration of a fluid element subject to any particular set of forces. We shall neglect this below.

The Navier Stokes equation is coupled to the nonlinear advection-diffusion equation ( $c f$. Appendix A for a derivation)

$$
\frac{\partial \hat{\phi}}{\partial t}+\mathbf{u} \cdot \nabla \hat{\phi}=\nabla(\bar{D} f(\hat{\phi}) \nabla \hat{\phi})+(1-\hat{\phi}) \xi
$$

The comoving derivative on the left accounts for 'advection': the process whereby composition is transported by bulk fluid flow. The first term on the right represents diffusive currents, and the second is a source term for the supersaturation $\hat{\phi}$. In contrast to the linear diffusion equations appearing in other settings, this equation has two remarkable features: 
(i) The variable $\xi(t)$ denotes $\phi_{0}^{-1} d \phi_{0} / d t$ and is a measure of the ramp rate. The source term, in which $\xi$ appears, characterizes the change of composition $\phi_{0}(T)$ with time during the temperature ramp, which gives rise to a constant increase of the relative supersaturation $\hat{\phi}$ under conditions where no diffusion or advection take place. As far as $\hat{\phi}$ is concerned, the effect of the temperature ramp is therefore to create supersaturation, at a rate controlled by $\xi$, throughout the sample.

(ii) Equation 3.2 involves an effective nonlinear diffusivity $\bar{D} f(\hat{\phi})$, which decreases with the relative supersaturation $\hat{\phi}$, becoming negative for $\hat{\phi}>\hat{\phi}_{s}$ (Bray 1994). In particular $\bar{D}$ is chosen in such a way that $f(0)=1$ and $f\left(\hat{\phi}_{s}\right)=0$ at the spinodal $\hat{\phi}_{s}$ where any state of uniform $\hat{\phi}>\hat{\phi}_{s}$ becomes locally unstable. Henceforth, we assume, for simplicity, that $\bar{D}$ and $f(\hat{\phi})$ do not depend directly on temperature, so that the only $T$-dependence of the diffusive dynamics enters parametrically through the definition, $\hat{\phi}=1-\phi / \phi_{0}(T)$, of the relative supersaturation.

\section{(b) Dimensionless parameters}

The equations of motion can be made dimensionless by introducing suitable scales of mass, length and time. As a length scale we choose the height of the sample $\Lambda \equiv \Lambda_{z}$ as the unit of length, and $\Lambda^{2} / \bar{D}$ as time unit. A mass unit is $\rho \Lambda^{3}$ but interestingly, none is really needed. The fluid density in equation 3.1 factors through all terms in the equation, except for the one involving the pressure, $\nabla(p / \rho+g z)$, whose sole purpose is to maintain incompressibility. We will write this term as a dimensionless gradient $\nabla \tilde{p}$, but could equally well eliminate it by projecting the Navier Stokes equation onto incompressible flows in a standard fashion (see e.g. Onuki 2002).

With these units, allowing for the various assumptions outlined above, and furthermore imposing a constant 'ramp rate' $\xi$, we find that the equations of motion take the following form:

$$
\begin{aligned}
& \frac{\partial \mathbf{u}}{\partial t}+\mathbf{u} \cdot \nabla \mathbf{u}=N_{1}\left[\nabla^{2} \mathbf{u}+\left(N_{0} / N_{2}\right) \hat{\phi} \nabla z-\nabla \tilde{p}\right] \\
& \frac{\partial \hat{\phi}}{\partial t}+\mathbf{u} \cdot \nabla \hat{\phi}=\nabla(f(\hat{\phi}) \nabla \hat{\phi})+(1-\hat{\phi}) N_{2}
\end{aligned}
$$

with three dimensionless parameter groups:

$$
N_{1}=\nu / \bar{D} \quad ; \quad N_{2}=\xi \Lambda^{2} / \bar{D} \quad ; \quad \frac{N_{0}}{N_{2}}=\alpha g \phi_{0} \Lambda^{3} / \bar{D} \nu
$$

Of these, $N_{1}$ is a material parameter of the binary fluid (the ratio of momentum to particle diffusivities). $N_{2}$ can be thought of as a dimensionless ramp rate (although a different one, which does not depend on $\Lambda$, will be introduced later on). The group we have denoted $N_{0} / N_{2}$ is, in the same sense, a dimensionless gravity parameter. But we will find below that the product of this with $N_{2}$ plays a special role in the theory; that product deserves its own name, and we call it $N_{0}=\alpha g \phi_{0} \xi \Lambda^{5} / \bar{D}^{2} \nu$. Although these three groups can be combined in numerous ways to create new dimensionless numbers, they are sufficient to fully describe the parameter space in our idealized problem, for a given sample shape and given $f(\hat{\phi})$.

The statement that they are sufficient assumes that no important physics has been left out of the equations of motion 3.3 and 3.4. Perhaps the strongest candi- 
dates for missing physics are (a) thermal gradients, which we have neglected from the outset; and (b) interfacial tension, which arise locally once the nonlinear regime of droplet formation is encountered, and are present at all times at the meniscus between phases. (Appendix A describes how a tension could be included.)

One dangerous-looking combination of these neglected effects is when interfacial tension and thermal gradients combine to create Marangoni stresses (that is, spatial gradients of the tension); these are known to be implicated in several interfacial instabilities (Sternling \& Scriven 1959, Davis 1987). While it would be imprudent to rule out an important role for Marangoni stresses and other thermal-gradient related effects, we do think it worth neglecting these in the first instance.

\section{(c) Relation to a Bénard problem: Advective instability}

Consider first the limiting case where $f(\hat{\phi})=1$, or equivalently, where $\hat{\phi}$ remains infinitesimal. Once the meniscus has formed, we can envisage a static diffusive state in which a steady current of supersaturation flows from the upper phase towards the lower and vice versa. (Note that, in such a state, $\hat{\phi}$ vanishes on the meniscus itself.) This current arises from the source term $N_{2}$ in equation 3.4, which creates supersaturation uniformly through space. However, were such a current to come from a localized source that maintained constant supersaturation $\hat{\phi}^{*}$ at the top of the sample, equations 3.3 and 3.4 would be isomorphic to those for the standard (Rayleigh-)Bénard problem (see e.g., Faber 1995). The latter concerns a single phase fluid heated from below; this creates an inverted density gradient (with the denser fluid on top) so that above a certain heating rate the system becomes unstable. The role of the local temperature field, in the Bénard problem, is played in ours by the supersaturation; that of the temperature difference between the plates, by $\hat{\phi}^{*}$. The correspondence applies whatever the sign of the expansion coefficient $\alpha$ : the less dense phase is always on top, so that supersaturation in this phase, which is largest far from the meniscus, always leads to a density inversion (and the same happens, symmetrically, in the lower phase too).

The presence of a distributed source term, rather than driving at the boundary, gives an extra complication to this analogy due to the nontrivial form of the quiescent profile. This strongly alters the details of any stability analysis, but not the basic ideas. First, note that because the Bénard problem is linear in the diffusive sector, it is governed by only two dimensionless groups, not three. This is the case in our problem also, so long as $\hat{\phi}$ (controlled by $N_{2}$ ) remains small enough. The two parameters normally chosen for the Bénard problem are the Prandtl number, which is the direct analogue of our $N_{1}$, and the Rayleigh number, which in our language is $\mathrm{Ra}=\left(N_{0} / N_{2}\right) \hat{\phi}^{*}$. The latter expression pertains to the 'standard' case of a constant supersaturation $\hat{\phi}^{*}$ at the upper boundary. When the source is distributed uniformly, this characteristic supersaturation scale instead depends on both the ramp rate and the system size, as $\hat{\phi}^{*} \simeq \xi \Lambda^{2} / \bar{D}=N_{2}$. This follows from the linearized diffusion equation: $\hat{\phi}^{*}$ is the supersaturation that builds up at a distance of order $\Lambda$ from the meniscus within the time it takes for diffusive relaxation over that distance. Combining these results, we may therefore identify $N_{0}$ itself as the direct analogue of a Rayleigh number for our variant of the Bénard problem.

Much is known about the Bénard problem, and using it we may now guess, with reasonable confidence, what will happen as the ramp rate $\xi$ is increased. The static 

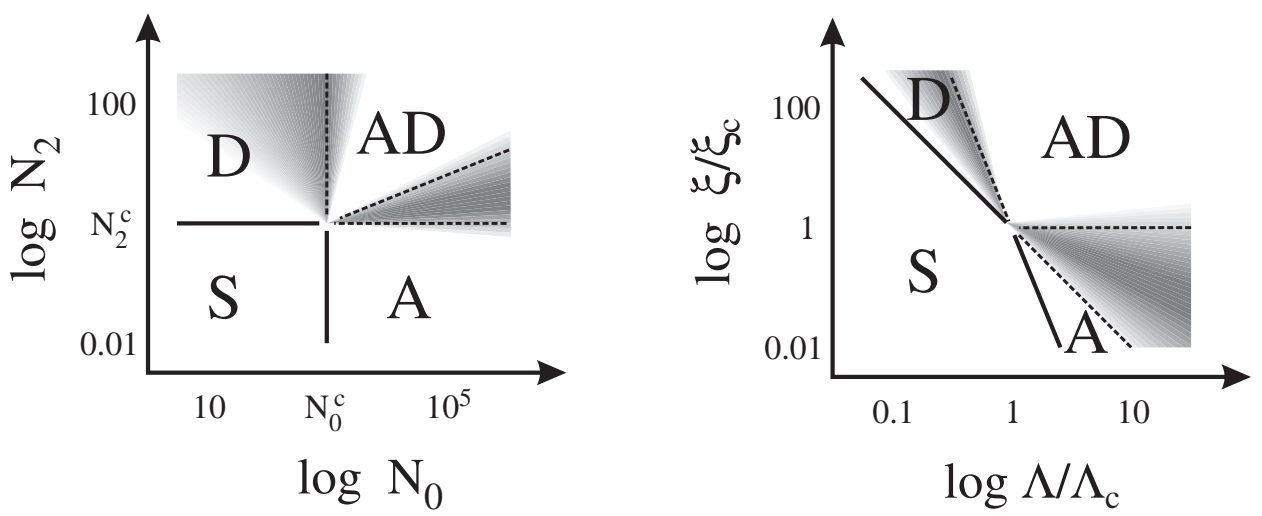

Figure 2. Suggested schematic phase diagram for an isothermally ramped binary fluid. The left panel gives the phase diagram in terms of the dimensionless control parameters $N_{0}$ and $N_{2}$, while the right is given in terms of $\Lambda$ and $\xi$; both in $\log / \log$ plot. The regions with qualitatively different behaviour are marked as $\mathrm{S}=$ stable, $\mathrm{A}=$ advectively unstable, $\mathrm{D}=$ diffusively unstable (perhaps with steady sedimentation), $\mathrm{AD}=$ advectively and diffusively unstable. Crossover lines from $\mathrm{D}$ to $\mathrm{AD}$ and from $\mathrm{A}$ to $\mathrm{AD}$ probably lie within the shaded regions shown. (In practice, of course, the boundary of the $\mathrm{S}$ region will not really have a sharp corner but will be a smooth curve, with the straight lines shown here as asymptotes. Likewise the 'crossover lines' need not meet the phase boundary precisely at this corner.)

diffusive profile will be stable until $N_{0}$ exceeds a critical value $N_{0}^{c}$ (for the standard Bénard problem, this is about 1700); thereafter, circulating fluid rolls will arise. This transition is represented in the phase diagram Figure 2 (left) as the transition from the region marked $\mathrm{S}$ to $\mathrm{A}$. As $N_{0}$ increases further, these rolls will undergo a series of bifurcations through various steady and perhaps unsteady (including oscillatory) states, with the details of this process controlled by $N_{1}$. Eventually at some much larger $N_{0}$ (also dependent on $N_{1}$ ), chaotic motion will set in, leading finally to fluid turbulence.

Note in passing that, although the rolls in Bénard are normally called 'convection rolls', they are properly 'advection rolls', in the following sense. The onset of these rolls is controlled by the advective nonlinearity (transport of supersaturation by fluid, which is the $\mathbf{u} . \nabla \hat{\phi}$ term on the left side of equation 3.4) not the convective one (transport of momentum by fluid, which is the $\mathbf{u}$. $\nabla \mathbf{u}$ in equation 3.3 ). For typical fluid parameters $\left(N_{1} \simeq 1\right.$ or more) one must move rather far along the sequence of transitions beyond $N_{0}^{c}$ before the Reynolds number becomes large enough that convective, rather than advective, nonlinearity becomes important.

\section{(d) Onset of droplets: Diffusive instability}

All of the preceding subsection refers to the case where the characteristic supersaturation $\hat{\phi}^{*}$ remains small enough that the diffusion is linear. Let us now consider the opposite case where $N_{0}$ remains small, but $\hat{\phi}$ does not. 
For small $N_{0}$ we expect no onset of advection rolls before the diffusive nonlinearity kicks in. Within a linearized framework, we found $\hat{\phi}^{*} \simeq N_{2}$, so the onset of the diffusive nonlinearity, if present on its own, must be governed simply by $N_{2} \equiv \xi \Lambda^{2} / \bar{D}$. When this reaches some critical value $N_{2}=N_{2}^{c}$ (which in general depends on the shape of $f(\hat{\phi})$ ), the stationary diffusion profile becomes unstable, which is indicated in the phase diagram (Figure 2, left) by the transition from the region $\mathrm{S}$ to $\mathrm{D}$. In region $\mathrm{D}$ droplets are formed by either a spinodal or a nucleation process. The instability must occur at or before the point where the supersaturation in the static profile reaches $\hat{\phi}_{s}$; this yields an upper bound $N_{2}^{c} \leq N_{2}^{c, s}$ (at which the region of highest supersaturation is locally unstable to spinodal decomposition). A particular choice of $f$ yields $N_{2}^{c}=N_{2}^{c, s} \simeq 1$ at small enough $N_{0}$ ( $\mathrm{J}$ Vollmer, unpublished), which appears to be adequate for our present purposes. In physical terms this result states that the diffusive instability kicks in whenever the height of the sample exceeds the length scale $(\bar{D} \xi)^{1 / 2}$ beyond which diffusion can no longer compete with the homogeneous growth of supersaturation characterized by $\xi$.

It is not completely clear what should happen once droplet formation begins. If gravity is strictly zero $\left(N_{0}=0\right)$ the system will coarsen without ever forming a horizontal meniscus; the presence of the ramp may cause domain formation within existing domains, reminiscent of patterns seen in certain binary systems undergoing simultaneous phase separation and polymerization (Clarke et al. 1995). (This process might conceivably lead to oscillatory states; the details certainly depend on $N_{1}$.) In any case, once gravity is switched on, sedimentation of the nucleated domains will occur. By gathering supersaturation into localized droplets, nucleation is likely to result in the onset of advective nonlinearity sooner than would be the case without it; that is, before the line $N_{0}=N_{0}^{c}$ is crossed (gray region in sector D). In the first instance the result could merely be steady sedimentation of the droplets; although this is, technically, an 'advective nonlinearity' (since it involves the $\mathbf{u} . \nabla \hat{\phi}$ term in equation 3.4) it is relatively benign. By relaxing supersaturation, this could cause the threshold for bulk flow to move somewhat beyond $N_{0}^{c}$; but such sedimentation, once present, is liable to give rise to fronts (Russel et al 1989) and other collective nonlinear behaviour (Chaikin 2000), distinct from those of the Bénard problem. In summary, the onset of advective nonlinearity (beyond simple sedimentation), in a state where the diffusive one is already active, should occur at a threshold which is likely to lie somewhere between the diffusive instability line $\left(N_{2}=N_{2}^{c}\right)$ and the continuation of the advective instability line $\left(N_{0}=N_{0}^{c}\right)$.

By the same token, starting with $N_{0}>N_{0}^{c}$ and $N_{2}$ small, the presence of Bénard rolls will clearly alter the criterion for onset of droplet formation. Here one can argue that the Bénard rolls are more efficient than pure diffusion at transporting supersaturation, so that the onset of the diffusive instability will be delayed beyond the line $N_{2}=N_{2}^{c}$ as was calculated for small $N_{0}$. On the other hand, for a given ramp rate $\xi$ (and fixed material parameters), we can find the cell size $\Lambda^{*}=\Lambda\left(N_{0}^{c} / N_{0}\right)^{1 / 5}$ for which the Bénard instability would be just incipient. It would be surprising if, in a sample of size $\Lambda \gg \Lambda^{*}$, the supersaturation far from the meniscus were actually less (for the same ramp rate and material parameters) than the value $\hat{\phi}^{*} \simeq$ $N_{2}\left(\Lambda^{*} / \Lambda\right)^{2}$ pertaining to this smaller cell. This suggests that diffusive instability should set in at the latest upon achieving the condition $N_{2}\left(N_{0}^{c} / N_{0}\right)^{2 / 5}=N_{2}^{c}$, where the left side of the equation is the value of $N_{2}$ in a fictitious sample of height 
$\Lambda^{*}$. In summary, the onset of diffusive nonlinearity, in a state where the advective one is already active, should occur somewhere in the shaded region marking the transition from the $\mathrm{A}$ to the $\mathrm{AD}$ regions in the left diagram of Figure 2, whose boundaries are the continuation of the diffusive instability line $\left(N_{2}=N_{2}^{c}\right)$ and the line $N_{2} / N_{2}^{c} \sim\left(N_{0} / N_{0}^{c}\right)^{2 / 5}$.

\section{(e) Nonequilibrium phase diagram for experimental control parameters}

The above arguments, which were summarized in the left panel of Figure 2, predict a phase boundary between a stable diffusive regime $(\mathrm{S})$ and an advectively unstable one (A) at $N_{0}=N_{0}^{c}$; and a boundary between $\mathrm{S}$ and a diffusively unstable regime (D) at $N_{2}=N_{2}^{c}$. For comparison with experiment it is helpful to represent these lines on a plot that involves two more accessible parameters, the system size $\Lambda$ and ramp rate $\xi$, as the horizontal and vertical axes (Figure 2, right). To this end we observe that the combinations $N_{0} / N_{2}=\left(\Lambda / \Lambda_{0}\right)^{3}$ and $N_{2}^{5} / N_{0}^{2}=\left(\xi / \xi_{0}\right)^{3}$ only depend on material constants. In these expressions we have identified 'natural' units of length and heating rate $\Lambda_{0}^{3}=\bar{D} \nu / \alpha g \phi_{0}$ and $\xi_{0}^{3}=\alpha^{2} g^{2} \phi_{0}^{2} \bar{D} / \nu^{2}=\left(\bar{D} / \Lambda_{0}^{2}\right)^{3}$, respectively, only depend on material constants. Consequently, on logarithmic scales the phase diagrams can be related by the linear transformation

$$
\left(\begin{array}{l}
\log \frac{\Lambda}{\Lambda_{c}} \\
\log \frac{\xi}{\xi_{c}}
\end{array}\right)=\left(\begin{array}{rr}
\frac{1}{3} & -\frac{1}{3} \\
-\frac{2}{3} & \frac{5}{3}
\end{array}\right)\left(\begin{array}{l}
\log \frac{N_{0}}{N_{0}^{c}} \\
\log \frac{N_{2}}{N_{2}^{c}}
\end{array}\right)
$$

where $\Lambda_{c}$ and $\xi_{c}$ are chosen so that the two boundaries of the stable region $\mathrm{S}$ intersect at $(1,1)$, i.e., the sharp corner visible in the right panel of Figure 2. Consequently, the S/A and S/D lines now meet at the point $\left(\Lambda_{c}, \xi_{c}\right)$, where $\Lambda_{c} / \Lambda_{0}=\left(N_{0}^{c} / N_{2}^{c}\right)^{-1 / 3}$, and $\xi_{c} / \xi_{0}=\left(N_{2}^{c}\right)^{5 / 3}\left(N_{0}^{c}\right)^{-2 / 3}$ as defined previously.

The estimates for the boundaries between the stable domain $\mathrm{S}$ and the advectively (A) and diffusively (D) unstable ones translate in this phase diagram into straight lines with slope -5 and -2 , respectively. The limiting estimates made above for where regimes $\mathrm{A}$ and $\mathrm{D}$ should each cross into $\mathrm{AD}$ (the regime in which advective and diffusive nonlinearities are simultaneously strong) are shown by dashed lines as previously, and the uncertainties for these regions are again indicated by gray areas. The estimate of the upper bound for the appearance of nucleation in the advective regime $N_{2} \sim N_{0}^{2 / 5}$ translates into a condition on the ramp rate $\xi$, and is independent of the sample height $\Lambda$ (although it will, like other critical parameters, in general depend on the shape of the sample cell, and on $f(\hat{\phi}))$.

Not shown in either phase diagram are additional, increasingly vague crossovers from $\mathrm{A}, \mathrm{D}$ and $\mathrm{AD}$ into further regimes where the convective nonlinearity also becomes important.

\section{Oscillation Mechanism}

In the experiments showing oscillatory demixing in $\mathrm{C}_{4} \mathrm{E}_{1}$, that were outlined in $\S 2$, one observes fluid flow (without obvious signs of turbulence) and droplet formation simultaneously. This means that advective and diffusive nonlinearities are both involved, making it plausible that these experiments lie within the $\mathrm{AD}$ region of the phase diagram. Preliminary parameter estimates (Vollmer et al., unpublished) 
suggest that they may lie in a part of the $\mathrm{AD}$ region well to the right of, but not much above, the point $\left(\Lambda_{c}, \xi_{c}\right)$ on Figure 2 (right). However, in the experiment it is $d T / d t$ that is held constant, not $\xi$, which can therefore drift slowly during the ramp, as can $\alpha, \nu$, etc. These drifts may cause the experimental parameters to move gradually towards more stable values, causing the oscillations finally to cease.

These remarks do not explain why the oscillations are there in the first place. Elsewhere we propose that in this part of the AD region, advection rolls form soon after creation of the meniscus, while the supersaturation $\hat{\phi}$ is still small. These rolls do not advect enough flux to maintain $\hat{\phi}$ below the threshold for droplet nucleation. Hence droplets appear; they then grow for a time, but before long, sedimentation sets in. The falling droplets soak up the remaining supersaturation, restoring the system to a state of small $\hat{\phi}$, so that the cycle is ready to begin again. In Vollmer et al. (unpublished) we find this scenario consistent with preliminary computer simulation data, and broadly in line with experimental data on various systems.

This explanation has something in common with a proposed mechanism for rhythmic deposition of precipitate in a convecting magma chamber cooled from above (Sparks et al. 1993), a problem that seems, however, to involve a significantly larger parameter space than the model considered here.

\section{Conclusion}

Even within the idealized model presented here, the interplay of gravity-driven advection and nonlinear diffusion presents an interesting theoretical challenge for the description of binary fluid demixing in the presence of a temperature ramp. The experimentally observed oscillations (Vollmer et al. 2002) appear in a regime where both nonlinearities are simultaneously large; this makes analysis difficult. Detailed computer simulations, now planned, may be a better way of finding out whether the physical ingredients retained in the idealized model (equations 3.3 and 3.4) are sufficient to explain the observed oscillations, or whether factors that we have omitted, such as Marangoni stresses arising from thermal gradients, play an essential role in this problem.

J.V. acknowledges financial support from the Schloessmann Foundation of the Max Planck Society, and thanks Howard Stone and Michael Brenner for useful discussions. D. Vollmer thanks the EC for a Marie Curie Fellowship.

\section{Appendix A. Advection-diffusion equation for the supersaturation $\hat{\phi}$}

The evolution of the composition $\phi$ of the mixture is governed by the advectiondiffusion equation

$$
\frac{\partial \phi}{\partial t}+\mathbf{u} \cdot \nabla \phi=-\nabla J_{\phi}
$$

where the comoving derivative on the left accounts for the advection of composition by the bulk fluid flow; and the divergence of the diffusive current $J_{\phi}$ on the right hand side accounts for the diffusive decay of composition gradients. The current $J_{\phi}=-M \nabla \mu$, with $M$ a mobility, is driven by the gradient of chemical potential $\mu$, which - in local equilibrium - obeys $\mu=\delta F / \delta \phi$, where a suitable choice for the 
free energy functional $F[\phi]$ is

$$
F[\phi]=\int \mathrm{d} V\left[F_{0}(\phi)+\kappa(\nabla \phi)^{2}\right]
$$

This involves the free energy density $F_{0}(\phi)$ describing homogeneous phases in equilibrium, and an energetic penalty $\kappa(\nabla \phi)^{2}$ for steep changes of composition.

For nonvanishing $\kappa$, the free energy functional A 2 supports static interfaces between phases which have small but finite width, and gives an equilibrium interfacial tension that depends on $\kappa$ and $F_{0}(\phi)$ (Bray 1994, Onuki 2002). Although interfacial tension can thus be included without much modification into the equations of motion, this would introduce a fourth dimensionless control parameter into our model. We assume here that it plays no major role and omit the corresponding terms from the equations of motion, although in our preferred simulation algorithm for this type of problem (lattice Boltzmann; see Kendon 2001), they are actually incorporated in precisely the above way.

Evaluating A 1 with $\kappa=0$ and $J_{\phi}=-M \nabla(\delta F / \delta \phi)$, introducing the abbreviations $\bar{D} \equiv M\left[\delta^{2} F_{0}[\phi] / \delta \phi^{2}\right]_{\phi=\phi_{0}}, f(\phi) \equiv(M / \bar{D}) \delta^{2} F_{0}[\phi] / \delta \phi^{2}$, and taking into account the temperature dependence of the factor $\phi_{0}$ appearing in the dimensionless supersaturation $\hat{\phi}$ one obtains equation 3.2 for the evolution of $\hat{\phi}$.

\section{References}

Aarts, D. G. A. L.; van der Wiel, J. H. \& Lekkerkerker, H. N. W. 2002 Interfacial dynamics and the static profile near a single wall in a model polymer mixture. J. Phys. Cond. Matt. **, ***_***.

Brandt, A. \& Fernando, H. J. S. (eds) 1995 Double diffusive convection. AGU Geophysics Monograph Series, Washington DC.

Bray, A. J. 1994 Theory of phase-ordering kinetics. Adv. Phys. 43 357-459.

Bray, A. J. 2000 Coarsening dynamics of nonequilibrium phase transitions. In Soft and fragile matter, nonequilibrium dynamics, metastability and flow (eds Cates, M. E. \& Evans, M. R.) IOP Publishing, Bristol.

Chaikin, P. M. 2000 Thermodynamics and hydrodynamics of hard spheres: the role of gravity. In Soft and fragile matter, nonequilibrium dynamics, metastability and flow (eds Cates, M. E. \& Evans, M. R.) IOP Publishing, Bristol.

Chan, C. K. \& Goldberg, W. I. 1987 Late-stage phase separation and fluid flow in a binary liquid mixture. Phys. Rev. Lett 58, 674-677.

Clarke, N., McLeish, T. C. B \& Jenkins, S. D. 1995 Phase-behavior of linear branched polymer blends. Macromolecules 28, 4650-4659.

Davis, S. H. 1987 Thermocapillary instabilities. Ann. Rev. Fluid Mech. 19, 403-435.

Faber, T. E. 1995 Fluid mechanics for physicists, Cambridge University Press, Cambridge.

Kendon, V. M.; Cates, M. E., Pagonabarraga, I., Desplat, J.-C. \& Bladon, P. 2001 Inertial effects in three-dimensional spinodal decomposition of a symmetric binary fluid mixture: a lattice Boltzmann study. J. Fluid Mech. 440, 147-203.

Onuki, A. 2002 Phase transition dynamics, Cambridge University Press, Cambridge.

Russel, W.B., Saville, D.A. \& Schowalter, W.R. 1989 Colloidal Dispersions, Chapter 12, Cambridge University Press, Cambridge.

Sternling, C. V. \& Scriven, L. E. 1959 Interfacial turbulence: Hydrodynamic instability and the Marangoni effect. A.I.Ch.E. J. 5, 514-523. 
Sparks, R. S., Huppert, H. E., Koyaguchi, T. \& Hallworth, M. A. 1993 Origin of modal and rhythmic igneous layering by sedimentation in a convecting magma chamber. Nature 61, 246-249.

Vollmer, D., Vollmer J. \& Wagner A. J. 2002 Oscillatory kinetics of phase separation in a binary mixture under constant heating. Phys. Chem. Chem. Phys. 4, 1380-1385. 